\title{
Atuação do Fisioterapeuta na saúde primária no enfrentamento da COVID19: relato de experiência
}

\section{Primary care physiotherapists' performance in the fight against COVID19: an experience report}

\section{Actuación del fisioterapeuta en la atención primaria de salud para el afrontamiento de la COVID19: relato de experiencia}

\author{
Eduardo Augusto Barbosa Figueiredo iD \\ Universidade Federal dos Vales do Jequitinhonha e Mucuri - Diamantina (MG) - Brasil
}

Keity Lamary Souza Silva iD

Universidade Federal dos Vales do Jequitinhonha e Mucuri - Diamantina (MG) - Brasil

Hiago Daniel Herédia Luz iD

Universidade Federal dos Vales do Jequitinhonha e Mucuri - Diamantina (MG) - Brasil

Fábio Luiz Mendonça Martins iD

Universidade Federal dos Vales do Jequitinhonha e Mucuri - Diamantina (MG) - Brasil

Márcio Alves Marçal (iD

Universidade Federal dos Vales do Jequitinhonha e Mucuri - Diamantina (MG) - Brasil

Débora Fernandes de Melo Vitorino iD

Universidade Federal dos Vales do Jequitinhonha e Mucuri - Diamantina (MG) - Brasil

Henrique Silveira Costa

Universidade Federal dos Vales do Jequitinhonha e Mucuri - Diamantina (MG) - Brasil

\section{RESUMO}

Objetivo: Relatar a experiência de residentes de fisioterapia em saúde coletiva no enfrentamento multidisciplinar e desenvolvimento de ações frente à coronavirus disease 2019 (COVID-19). Síntese dos dados: Trata-se de um relato de experiência a partir da vivência prática dos residentes, em decorrência da pandemia da COVID19, em duas cidades, Datas e Presidente Kubitschek, no interior de Minas Gerais, Brasil. As atividades ocorreram de março a junho de 2020. As ações foram realizadas por meio de orientações, roda de conversa, atendimento por videochamadas e fôlderes. As propostas sintetizaram: Ações de prevenção à saúde; Atenção à saúde mental e física dos trabalhadores da saúde; Educação em saúde aos trabalhadores de outros setores das prefeituras; Monitoramento de pacientes crônicos; e Educação permanente dos agentes comunitários de saúde. As atividades colaboraram com a prática clínica dos residentes, permitindo a construção com a equipe de saúde de propostas para um enfrentamento seguro no manejo da COVID19. Conclusão: As propostas elaboradas no início da pandemia permitiram realizar ações que auxiliaram a atuação da equipe de Saúde da Família no enfrentamento à COVID19. A experiência dos residentes contribuiu para a construção dos saberes e da experiência de gestão no enfrentamento da pandemia, colaborando para a formação dos residentes.

Descritores: Infecções por Coronavirus; Saúde Pública; Educação em Saúde; Fisioterapia.

\section{ABSTRACT}

Objective: To report on the experience of public health physiotherapy residents in the multidisciplinary fight and development of actions against the coronavirus disease 2019 (COVID-19). Data synthesis: This is an experience report on the practical work develop by the residents during the COVID-19 pandemic in two cities, Datas and Presidente Kubitschek, located in countryside of Minas Gerais, Brazil. The activities took place from March to June 2020. The activities consisted of guidelines, conversation circles, video calls, and flyers. The proposals summarized: Disease prevention actions; Health workers' mental and physical health care; Health Education for workers in other sectors of city halls; Monitoring of chronic patients; and Permanent education

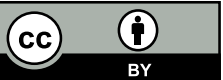


of community health workers. The activities contributed to the residents' clinical practice and allowed them to partner up with the health care team for the development of proposals for safely tackling and managing COVID-19. Conclusion: The proposals developed at the beginning of the pandemic allowed taking action to help the Family Health Team to tackle COVID-19. The residents' experience contributed to the development of knowledge and management experience in tackling the pandemic, thus contributing to the residents' training.

Descriptors: Coronavirus Infections; Public Health; Health Education; Physical Therapy Specialty.

\section{RESUMEN}

Objetivo: Relatar la experiencia de residentes de fisioterapia en salud colectiva para el afrontamiento multidisciplinar y el desarrollo de acciones contra el coronavirus disease 2019 (COVID-19). Síntesis de los datos: Se trata de un relato de experiencia a partir de la práctica de los residentes decurrente de la pandemia de la COVID19, en dos ciudades, Datas y Presidente Kubitschek del interior de Minas Gerais, Brasil. Las actividades se dieron entre marzo y junio de 2020. Se realizaron las acciones a través de orientaciones, rueda de conversación, atención por video-llamadas y folletos. Las propuestas sintetizaron: Acciones de prevención a la salud; Atención a la salud mental y física de los trabajadores sanitarios; Educación en salud para los trabajadores de otros sectores de los ayuntamientos; Monitoreo de pacientes crónicos; y Educación permanente de los agentes comunitarios de salud. Las actividades colaboraron con la práctica clínica de los residentes permitiendo la construcción de propuestas para el afrontamiento seguro del manejo de la COVID-19 con el equipo de salud. Conclusión: Las propuestas elaboradas en el principio de la pandemia permitieron la realización de acciones que ayudaron la actuación del equipo de Salud de la Familia para el afrontamiento de la COVID19. La experiencia de los residentes ha contribuido para la construcción de los saberes y de la experiencia de gestión para el afrontamiento de la pandemia contribuyendo para la formación de los residentes.

Descriptores: Infecciones por Coronavirus; Salud Pública; Educación en Salud; Fisioterapia.

\section{INTRODUÇÃO}

O novo coronavírus é um vírus zoonótico denominado SARSCoV2 (severe acute respiratory syndrome coronavirus 2), descoberto por cientistas chineses, que ocasiona, por meio de infecção viral, a doença denominada COVID19 (coronavirus disease 2019)(1), relatada pela primeira vez em Wuhan/China no final de dezembro de 2019 e que se espalhou rapidamente para outros países ${ }^{(1)}$. O sistema respiratório é um dos mais afetados pela doença, podendo também desenvolver manifestações sistêmicas, incluindo distúrbios neurológicos, cardiovasculares, gastrointestinais e musculoesqueléticos ${ }^{(1-3)}$. A COVID19 foi classificada como pandemia e declarada, em 30 de janeiro de 2020, como emergência de saúde pública pela Organização Mundial da Saúde ${ }^{(4)}$.

A manifestação clínica da COVID-19 é ampla, podendo variar desde ausência de sintomas à pneumonia e morte ${ }^{(2)}$. Quando há sintomas, pode ser caracterizada como síndrome gripal, com febre, tosse, dores musculares, fadiga, hemoptise, cefaleia, dispneia ${ }^{(1,2)} \mathrm{e}$, em casos graves, pode haver a síndrome respiratória aguda grave. Para detecção da COVID-19 é necessária a testagem por exames laboratoriais ou, quando esses não estão disponíveis, pode ser realizado diagnóstico clínico e epidemiológico, com os casos confirmados sendo obrigatoriamente notificados ${ }^{(1)}$.

O primeiro caso de COVID-19 no Brasil e na América do Sul(4) foi registrado em fevereiro de 2020, em São Paulo. O número de casos aumentou em decorrência da alta transmissibilidade ${ }^{(1)}$.

Os programas de residência multiprofissional na APS possuem o objetivo de ampliar a atuação da Estratégia de Saúde da Família (ESF) e o trabalho multiprofissional, pois o trabalho no Sistema Único de Saúde (SUS) exige novas configurações profissionais, que garantam a execução das diretrizes e princípios propostos para o funcionamento adequado do SUS ${ }^{(5)}$, devido à integralidade das ações. Entre os profissionais inseridos na APS, observa-se constante aumento do número de fisioterapeutas nas equipes ${ }^{(6)}$.

O fisioterapeuta, durante a graduação, adquire competências e habilidades que o possibilita agir em todos os níveis de atenção, no entanto, atualmente, tem havido uma maior preocupação com a formação de um profissional com perfil voltado para a APS. As diretrizes da ESF direcionam como competência dos profissionais de saúde, incluindo do fisioterapeuta, o desenvolvimento de ações e o desenvolvimento de serviços para tratamento e reabilitação e também atuação no controle dos riscos e danos em seu território, prevenindo agravos e promovendo a saúde com ações no âmbito individual, em grupos e em coletividade ${ }^{(7)}$.

Dessa forma, os profissionais de saúde necessitam da construção de competências e habilidades para exercerem essas mudanças ocorridas no setor da saúde e no paradigma de saúde pública. Por isso, tornam-se necessárias ações que envolvam práticas de saúde integrais, oportunas, contínuas e de qualidade para população(5). Assim 
sendo, a atuação dos residentes necessita ser integrada ao tripé "ensino-serviço-comunidade", por meio do diálogo existente entre preceptores universitários, a população e a gestão.

A pandemia da COVID-19 levou à reorganização dos processos de trabalho em todos os níveis de atenção à saúde. Na APS o trabalho baseia-se em frear a disseminação pelas características preventivas e promotoras à saúde relacionadas nesse âmbito da saúde ${ }^{(7)}$, visto que $80 \%$ dos casos de COVID19, considerados leves, serão manejados pelos profissionais da APS por ser a porta de entrada do SUS ${ }^{(7,8)}$.

Ressalta-se que os profissionais inseridos na APS devem desenvolver ações de combate e enfrentamento de doenças, segundo a Política Nacional de Promoção à Saúde ${ }^{(9)}$, ou seja, realizar ações que contribuam para a prevenção da contaminação pelo vírus e de possíveis agravos, a inserção da comunidade no contexto atual para combater a pandemia, com a equipe de saúde, para toda a população presente nos municípios durante esse período. Outro fator importante é atuar conforme o Plano de Contingência Nacional para Infecção Humana pelo novo Coronavírus COVID-19(10), com ações que variam desde vigilância até a gestão. No cenário atual da pandemia, é necessário promover ações e respostas rápidas e eficientes, especialmente nas Unidades Básicas de Saúde (UBS), principal responsável pela triagem e monitoramento dos casos suspeitos. Dessa forma, a estreita relação entre as ações de enfrentamento à COVID-19 e a Atenção Primaria à Saúde (APS) são consideradas de alta relevância.

Diante desse contexto, a relevância deste estudo está em vivenciar a experiência como residentes de fisioterapia em saúde coletiva sobre as ações destinadas ao combate da infecção da COVID-19 na APS, almejando contribuir nas ações de medidas preventivas da doença, no direcionamento clínico dos profissionais atuantes, na integração com a equipe e a comunidade, e obter aprendizado de cada experiência descrita.

Portanto, o presente artigo tem como objetivo relatar a experiência de residentes de fisioterapia em saúde coletiva no enfrentamento multidisciplinar e desenvolvimento de ações frente à coronavirus disease 2019 (COVID-19).

\section{SÍNTESE DE DADOS}

Trata-se de um relato de experiência obtido por meio das ações de enfrentamento à COVID-19 vivenciadas pelos residentes de fisioterapia em saúde coletiva de uma Universidade Federal, localizada na região do Vale do Jequitinhonha, que possui como macrorregião de saúde a cidade de Diamantina, Minas Gerais, Brasil, sendo as cidades de Datas e Presidente Kubitscheck pertencentes a essa macrorregião. As ações foram realizadas no período de março a junho de 2020, com a participação dos residentes, preceptores universitários e os respectivos centros de operações de emergência para reposta ao novo coronavírus de cada município, como previsto pelo Ministério da Saúde, para uma gestão coordenada, em reposta ao plano de contingência nacional, composta pelos gestores em saúde (secretário(a) municipal de saúde, coordenador(a) da atenção primária, equipe de profissionais multidisciplinares e conselheiros municipais de saúde), que desenvolveram ações de promoção e prevenção da saúde para a comunidade e os trabalhadores da prefeitura, apoio à saúde mental e física dos profissionais de saúde, além de capacitação dos agentes comunitários para atualização diante da evolução da COVID19 nos municípios.

As condutas consistiram em: Ações de prevenção à saúde; Atenção à saúde mental e física dos trabalhadores da saúde; Educação em Saúde aos trabalhadores de outros setores das prefeituras; Monitoramento de pacientes crônicos; e Educação Permanente dos Agentes Comunitários de Saúde. As ações habituais dos grupos operativos precisaram ser suspensas como forma de prevenção de contágio.

\section{Ações de prevenção à saúde}

A principal forma de transmissão do coronavírus parece ser a mobilidade humana ${ }^{(11)}$, pois as pessoas assintomáticas e sintomáticas, ao transitarem entre regiões, transmitem o vírus por meio de secreção das gotículas de saliva sem nenhuma restrição(1). A partir disso, a instalação de barreiras sanitárias se tornou uma importante ação para frear a disseminação do vírus por meio da prevenção, e é considerada uma ferramenta potencialmente eficaz no monitoramento dos casos de COVID19 ${ }^{(11,12)}$.

Essa medida de proteção foi instaurada em ambos os municípios citados acima, contando com a atuação dos residentes para auxiliar no planejamento, que envolveu reunião com a equipe local para elaborar estratégias, por meio de discussão e debates de ideias, que visassem à prevenção e promoção de saúde pela disseminação de informação sobre o novo coronavírus. A metodologia para prevenção foi busca ativa direta, ou seja, veículos seriam abordados pelos profissionais presentes na barreira (todos profissionais da saúde). Nessa abordagem, haveria a solicitação para o condutor abaixar o vidro, momento em que o profissional de saúde, com as devidas medidas de proteção, se apresentava e fornecia as instruções disponíveis para prevenção da COVID19, preenchia o formulário de triagem com possíveis sinais e sintomas, além dos dados de identificação, de interesse da prefeitura, com as 
seguintes variáveis: idade, profissão, sexo e motivo de estar entrando no município, por meio de interrogatório ao condutor e, posteriormente, seria aplicado produto de desinfecção no veículo.

Cada veículo que atravessava a barreira recebia, por meio de informações orais e de fôlderes, instruções sobre o que era a doença COVID19, as formas de contágio e sua prevenção (com uso de máscaras, distanciamento social e lavagem frequente das mãos), informação sobre os sinais e sintomas clínicos, bem como o direcionamento do fluxo de atendimento caso houvesse a contaminação( ${ }^{(1)}$. Esses temas eram explanados pelos residentes de maneira clara, sucinta e em linguagem popular, reforçando as informações obtidas nos fôlderes educativos disponibilizados por cada município.

Pela atuação dos residentes de fisioterapia nas barreiras sanitárias foi possível identificar os viajantes que adentraram nos municípios, para visitar familiares, desempenhar serviços nos municípios ou outras atividades. Entretanto, para que o objetivo preventivo da transmissão do vírus através da colocação da barreira fosse atingido, foi importante realizar o controle e o monitoramento dos visitantes ou moradores, que pôde ser feito a partir do preenchimento do formulário durante a abordagem. Esses dados foram utilizados para monitoramento posterior por chamada telefônica, destinado ao segmento do estado de saúde geral de cada indivíduo, garantindo a segurança do município. Essa experiência foi considerada ímpar para os residentes, que nunca haviam passado por situação similar a essa.

As ligações telefônicas eram realizadas de sete a quatorze dias após o preenchimento das fichas, considerando que a transmissibilidade do vírus ocorre, em média, sete dias após a infecção ${ }^{(13)}$. Logo, esse monitoramento constante assegura ao município o rastreio precoce de possíveis casos. Caso fosse relatado algum sinal ou sintoma da COVID-19, como febre, tosse, dores musculares, fadiga, hemoptise, dor de cabeça ou dispneia ${ }^{(1,2)}$, a pessoa era orientada a procurar a Unidade Básica de Saúde responsável pela área de sua residência.

Além disso, os casos suspeitos (pessoas que apresentam algum sinal ou sintoma de infecção pela COVID-19, mas que não apresentam exame laboratorial)(13) eram monitorados a cada 48 horas para a observação de evolução dos sintomas e possível contágio. Em cada ligação, eram reforçadas as medidas preventivas (isolamento social, uso de máscaras e lavagem periódica das mãos $)^{(1)}$ e de etiqueta respiratória, protegendo-se com lenços individuais, e preferencialmente descartáveis, a boca e o nariz ao tossir e espirrar, para evitar a disseminação de gotículas (principal meio de contágio), ou na impossibilidade do lenço, cobrir com a região do cotovelo a face ${ }^{(14)}$, assim como qual fluxo de saúde deveria ser seguido conforme o Protocolo de Manejo Clínico do Coronavírus disponibilizado pelo Ministério da Saúde ${ }^{(7)}$.

Acredita-se que foi possível realizar um monitoramento seguro e eficaz, e que o auxílio dos residentes foi muito importante, tendo em vista que houve mais participantes na ação com os profissionais de saúde. A estratégia da barreira permitiu identificação e direcionamento precoce de casos suspeitos e manejo seguro, de acordo com o preconizado nacionalmente, sendo possível realizar promoção de saúde e prevenção de agravos.

\section{Atenção à saúde mental e física dos trabalhadores da saúde}

Os profissionais de saúde estão diretamente vinculados ao combate do coronavírus, potencializando sua chance de contágio. Dessa forma, a manutenção da saúde mental desses trabalhadores é um desafio no contexto da saúde coletiva, sendo necessário um olhar mais abrangente sobre o ambiente em que os trabalhadores estão inseridos ${ }^{(15)}$.

A proposta de atenção à saúde do trabalhador surgiu de uma demanda indicada pela Regional de Saúde de Diamantina. A partir disso, as Secretarias de Saúde dos respectivos municípios se reuniram com os residentes para planejarem em conjunto quais as possíveis soluções para a demanda. Em ambos os municípios, os planejamentos e atuações metodológicas divergiram, mas os dois utilizaram exercícios que estimulam o autoalongamento, ativação da circulação sanguínea e técnicas de padrões respiratórios.

No município de Datas, a atividade ocorreu no espaço externo da UBS, por possuir estrutura física e aparato técnico suficiente para distanciamento dos profissionais durante a atividade realizada de forma presencial. Já no município de Presidente Kubitschek, foi feita através de videochamadas, com salas criadas por meio de aplicativos, duas vezes por semana, com duração de cinquenta minutos e a contabilização dos participantes. Todas as atividades realizadas foram planejadas e marcadas com antecedência, sendo fornecido o endereço de acesso aos que demonstravam interesse em participar, assim fazendo com que houvesse uma maior adesão e participação.

Estar atento às condições psicológicas e físicas dos trabalhadores da saúde, principalmente nesse momento de pandemia, tornou-se de extrema importância, pois oportunizou elaborar medidas individuais e coletivas para reduzir os efeitos negativos do trabalho para esses profissionais ${ }^{(15)}$.

A atividade física é uma estratégia positiva no âmbito da saúde mental, pois, além de melhorar a dinâmica corporal e induzir o relaxamento ${ }^{(16)}$, tem sido amplamente discutida como intervenção positiva à saúde mental dos 
indivíduos, pois melhora a participação social, possui benefícios corporais, aumenta a qualidade de vida e ainda minimiza sinais e sintomas de distúrbios psiquiátricos, como ansiedade e depressão ou de doenças crônicas ${ }^{(17)}$.

Dessa forma, a participação dos residentes nessa ação promotora de saúde possibilitou que demonstrassem como o exercício é um aliado para saúde corporal e causa de bemestar. Ressalta-se que houve uma boa adesão, com aumento no número de participantes com o passar dos dias e um retorno positivo dos profissionais, e essa ação permitiu o fortalecimento e estreitamento do vínculo com a equipe de saúde.

\section{Educação em saúde aos trabalhadores de outros setores das prefeituras}

A educação em saúde é uma importante ferramenta que deve orientar a prática de saúde dos profissionais e tem por objetivo desenvolver capacidades em âmbito coletivo sobre a melhora das condições de vida de uma comunidade $^{(18)}$. Diante disso, durante reunião em equipe, criada para enfrentar a COVID19, foi estabelecida a necessidade de realizar ações de educação em saúde com os trabalhadores do setor de limpeza urbana e reciclagem do município de Presidente Kubitschek para sanar dúvidas referentes ao coronavírus, como a forma de contágio, prevenção e medidas de autocuidado, pois o serviço de limpeza urbana é essencial para a saúde humana e ambiental e contribui para auxiliar na prevenção e transmissão do coronavírus ${ }^{(19)}$, visto que o combate à pandemia requer ações intersetoriais ${ }^{(20)}$.

Para que essa abordagem fosse aceita pelos trabalhadores, a dinâmica de roda de conversa foi escolhida, pois permitiu abertura de espaço de diálogos e interações dos participantes, ampliando as percepções acerca do assunto e permitindo a reflexão das manifestações apresentadas ${ }^{(21)}$. A ação foi realizada no prédio da prefeitura municipal, na sala de limpeza urbana e na usina de reciclagem, fora do horário de trabalho dos servidores, contando com a participação de todos os funcionários do setor. Nessa roda de conversa, as discussões também foram direcionadas para orientar sobre os possíveis ajustes que poderiam ser realizados durante o processo de trabalho, minimizando possibilidade de contágio durante o serviço e de contaminação dos familiares. O uso correto de equipamentos de proteção individual (EPI) ${ }^{(15)}$, o acondicionamento de máscaras e a sugestão de uniforme específico para o trabalho, além de um local de higiene dos profissionais antes de retornarem aos domicílios.

Em seguida, a equipe de saúde sentiu a necessidade de agendar uma reunião com os gestores responsáveis por cada setor da limpeza pública, a fim de repassar essas mesmas informações que foram discutidas com os funcionários do setor. Acredita-se que, após a conversa com os servidores e gestores, o processo de trabalho pôde ser compreendido e adaptado por ambas as partes, além de ter estabelecido e ressaltado a importância de proteção dos trabalhadores para realizar o trabalho seguro diante a pandemia do coronavírus, proporcionando prevenção do contágio e promoção de saúde pela educação em saúde que foi construída com essa estratégia de debates e discussões dentro comunidade que se pretendia atingir.

O manejo seguro do lixo produzido requer a participação ativa da comunidade quando há casos suspeitos ou confirmados $^{(19)}$, pois muda o comportamento de geração de lixo infectante, requerendo identificação e manejo correto para minimizar as possibilidades de contágio em massa dos trabalhadores ${ }^{(20)}$. Em decorrência disso, houve também a elaboração de anúncios sobre a importância do manejo correto e indicado do lixo - como utilizar máscaras durante a separação dos resíduos, dedetizar e acondicionar em dois sacos plásticos identificados como "lixo infectado"(20), que foram reproduzidos por carros de som informantes e em fôlderes educativos a serem distribuídos para a população em toda área urbana, com a intenção de haver contribuição dos moradores da cidade no descarte correto do lixo (incluindo máscaras e luvas) no horário da coleta, bem como na separação do lixo reciclável do lixo comum, minimizando as chances de contágio dos trabalhadores da limpeza urbana e seus familiares.

Esse momento vivenciado pelos residentes evidencia um principio relevante do SUS, o princípio da intersetorialidade, um movimento de superação da visão e das práticas fragmentadas, sendo, ainda, uma estratégia de produção crescente de articulações entre diferentes segmentos, tanto no plano interno quanto no externo ${ }^{(22)}$.

Essas vivências podem orientar a resolução de alguns dos problemas da saúde, como esse caso dos trabalhadores da limpeza pública, pois o compromisso do setor saúde é tornar cada vez mais visível que o processo saúdeadoecimento é composto por múltiplos aspectos - pertinentes aos diversos setores governamentais, privados e não governamentais -, os quais devem compor suas agendas quando forem constituir suas ações e políticas específicas para garantia da saúde como direito humano e questão de cidadania ${ }^{(23)}$.

\section{Monitoramento de pacientes crônicos}

Os pacientes crônicos com condições pré-existentes, como hipertensão arterial sistêmica, diabetes mellitus, doença pulmonar obstrutiva crônica, cardiopatias e distúrbios circulatórios, além de idosos e imunodeprimidos, são 
classificados como grupos de risco e vulneráveis aos estágios avançados da COVID19, sendo necessária a redução da presença física desses usuários nos centros de saúde, optando preferencialmente pelo teleatendimento ${ }^{(24)}$.

Mas, em decorrência da precariedade socioeconômica dos municípios do Vale do Jequitinhonha, o teleatendimento é dificultado. Diante dessa realidade, estratégias de acompanhamento às pessoas com doenças crônicas foram repensadas e desenvolvidas no Centro de Operações de Emergências em Saúde Pública para o novo Coronavírus (https://portal.fiocruz.br/noticia/ministerio-da-saude-lanca-protocolo-de-tratamento-do-covid-19) a fim de minimizar a exposição desses usuários do sistema de saúde no ambiente extradomiciliar. Para isso, houve a criação de um roteiro de uso exclusivo da equipe dos possíveis sinais e sintomas clínicos que sugerem indícios de alterações clínicas da patologia pré-existente, para serem seguidos durante a visita, pois cerca de $90 \%$ dos casos de evento cardiovascular e outras condições de saúde demonstram sinais ou sintomas clínicos antes de agravarem, além de também contribuir na busca ativa dos sintomáticos respiratórios presentes na comunidade.

Durante a visita domiciliar, o fisioterapeuta residente colaborou com seu papel de agente transformador de saúde por meio da educação e informação, visto que é um profissional habilitado para trabalhar com prevenção de doenças gerais e maus hábitos que possam prejudicar a saúde do indivíduo ${ }^{(25)}$, uma vez que na cidade não havia, até o momento da ação, por meio de diagnóstico clínico ou exame laboratorial, confirmação da infeç̧ão pelo novo coronavírus. Em virtude disso, durante a visita, houve a divulgação e reforço das mensagens sobre a infecção pela COVID19 (prevenção de contágio, autocuidado e medidas de proteção comunitária) por todos os profissionais.

Esse plano de cuidado dos doentes crônicos ocorreu na visita domiciliar com orientação do ACS. As visitas eram realizadas conforme recomendações do Ministério de Saúde ${ }^{(24)}$, com distância entre as pessoas de dois metros, o uso de máscara e no ambiente extradomiciliar, obtendo respostas positivas no encaminhamento precoce dos pacientes com queixas clínicas, avaliação da interação medicamentosa e acompanhamento dos pacientes clinicamente estáveis.

O controle e as informações regulares permitiram obter um panorama do estado de saúde dos indivíduos. Assim, após a visita realizada nos domicílios de portadores de patologias pregressas, atualizou-se o número de telefone da residência ou do indivíduo (podendo ser também de algum responsável, caso necessário) para atualização dos dados e informações clínicas. A partir de então, com frequência regular, as pessoas recebiam ligações periódicas com o intuito de coletar informações acerca da patologia e da condição de saúde atual para um efetivo monitoramento, para, quando necessário, realizar o deslocamento de uma equipe capacitada ao domicílio ou orientá-los a procurar a ESF com horário marcado. Por meio da prevenção de agravos, com educação em saúde e reforço pelos usuários para a vigilância da atual condição de saúde, esse método permitiu aos residentes de fisioterapia compreender a importância do cuidado com os pacientes crônicos e o quão importante é a estabilidade clínica desses usuários.

\section{Educação permanente dos agentes comunitários de saúde}

Os agentes comunitários de saúdes (ACS) conhecem bem a comunidade onde trabalham, porém, como todo profissional da APS, devem ter capacitações para aprimoramento do processo de trabalho ${ }^{(26)}$, que também contribuem com a valorização de saberes prévios, a troca de experiências, o auxílio na identificação de situações na comunidade e influenciam positivamente o planejamento de ações ${ }^{(27)}$. Por meio da educação permanente é possível qualificar o profissional no processo de trabalho e permitir a construção de prática profissional segura ${ }^{(24)}$, que se faz necessária para permitir boa estruturação e atendimento à comunidade no trabalho dos $\operatorname{ACS}^{(27)}$.

Diante disso, após discussão com a equipe do Centro de Operações de Emergências em Saúde Pública para o novo Coronavírus, criou-se a proposta de capacitar os agentes de saúde, utilizando a educação permanente, para entender o processo de trabalho no contexto atual da pandemia da COVID19 e elucidar temas que pudessem trazer significância e despertassem o interesse dos agentes para o processo educativo. A partir disso, semanalmente, o grupo de ACS reunia-se com os residentes e a coordenadora da atenção primária, em uma roda de conversa, criando espaço para discussões e reflexões ${ }^{(21)}$ sobre conhecimentos acerca da promoção à saúde diante da pandemia do novo coronavírus. Os ACS eram estimulados a trazer na roda o que vivenciaram nas visitas acerca do coronavírus e, a partir desses casos, fluíam as conversas. Além disso, utilizava-se como material de apoio a cartilha do Ministério da Saúde com as recomendações que adequaram as ações dos ACS frente à atual situação epidemiológica referente à COVID19(28). A roda de conversa facilitou a discussão da cartilha e promoveu um ambiente acolhedor para que todos participassem ativamente da discussão e para que houvesse uma maior problematização da cartilha, pois este é um passo importante no processo de comunicação e permite uma maior troca de saberes e de experiências vivenciadas por meio da interação consigo próprio, com o outro e com o mundo(29).

Essa ação possibilitou o entendimento dos residentes de como são necessárias as discussões frequentes com os ACS, para entender os anseios e criar soluções com eles para que hajam respostas efetivas para as demandas 
criadas por eles e pelos usuários. Realizar o papel de colaborador nas capacitações contribuiu na resolução das demandas, além de permitir que houvesse o estreitamento da relação entre os fisioterapeutas residentes e os ACS, pois ambos criaram e compartilharam ideias em comum por meio de um diálogo eficaz. Viu-se a importância e a vivência enriquecedora da participação do fisioterapeuta na APS nesse momento da pandemia do novo coronavírus. Até o momento da finalização do presente estudo, a região do Vale do Jequitinhonha, onde estão localizadas as cidades de Datas e Presidente Kubitscheck, era uma das regiões com menor número de casos confirmados de COVID19 e a única no estado sem óbitos confirmados ${ }^{(30)}$.

Apesar do papel do fisioterapeuta não ser explícito na literatura de forma consolidada, até o ano de 2018 observou-se um crescente aumento da inserção desses profissionais na APS, por meio de estágios universitários, programas de residência e, principalmente, pelos Núcleos de Apoio à Saúde da Família( ${ }^{(6)}$. Portanto, reforça-se a contribuição qualificada desses profissionais no contexto da APS, porém cabe uma reflexão após a Nota Técnica n. ${ }^{0}$ 3/2020-DESF/SAPS/MS, que extingue e desvincula o NASF da atenção básica ${ }^{\left({ }^{11}\right)}$, do prejuízo e da dificuldade de inserção dos fisioterapeutas e de outros profissionais na APS, no contexto atual, abrindo espaço para um serviço insuficiente para a população, além de dificultar a realização dos princípios do SUS.

Sabe-se que a Política Nacional de Promoção da Saúde (PNPS) tem como objetivos principais promover a saúde e reduzir os riscos à saúde individual e da comunidade ${ }^{(9)}$. Nesse sentido, todas as experiências descritas em prol do trabalhador e da comunidade visaram atingir o objetivo principal da PNPS.

\section{CONCLUSÃO}

As ações realizadas pelos residentes, em parceria com os municípios estudados, permitiram a troca de saberes, a parceria entre a universidade e a gestão municipal, e apoio nesse momento delicado para a saúde coletiva, permitindo que o conhecimento teórico obtido na universidade pudesse, por meio da prática, ser vivenciado na comunidade com maior qualidade e segurança.

Reforça-se, ainda, a relevância da inserção do fisioterapeuta na equipe multiprofissional na APS, visto que as ações de prevenção e promoção de saúde foram elaboradas considerando a integração com a equipe multiprofissional, no diálogo com embasamento científico, contribuindo para a formação e a experiência dos residentes fisioterapeutas na educação em saúde.

\section{AGRADECIMENTOS}

Às secretarias municipais de saúde dos municípios de Datas e Presidente Kubitscheck, em Minas Gerais.

\section{CONTRIBUIÇÕES}

Eduardo Augusto Barbosa Figueiredo, Keity Lamary Souza Silva e Hiago Daniel Herédia Luz contribuíram com a aquisição, análise e interpretação dos dados; e a redação e/ou revisão do manuscrito. Fábio Luiz Mendonça, Márcio Laves Marçal, Débora Fernandes de Melo Vitorino e Henrique Silveira Costa contribuíram com a redação e/ou revisão do manuscrito.

\section{REFERÊNCIAS}

1. Ministério da Saúde (BR). Diagnóstico e Tratamento da COVID-19 [Internet]. 2020 [acesso em 2020 Ago 13]. Disponível em: https://sbim.org.br/images/files/notas-tecnicas/ddt-covid-19-200407.pdf

2. Wu D, Wu T, Liu Q, Yang Z. The SARS-CoV-2 outbreak: what we know. Int J Infect Dis [Internet]. 2020 [acesso em 2020 Ago 13];94:44-8. Disponível em: https://linkinghub.elsevier.com/retrieve/pii/ S1201971220301235

3. Liu PP, Blet A, Smyth D, Li H. The science underlying COVID-19. Circulation [Internet]. 2020 [acesso em 2020 Ago 13];142(1):68-78. Disponível em: https://www.ahajournals.org/doi/10.1161/ CIRCULATIONAHA.120.047549

4. Biscayart C, Angeleri P, Lloveras S, Chaves TSS, Schlagenhauf P, Rodríguez-Morales AJ. The next big threat to global health? 2019 novel coronavirus (2019-nCoV): What advice can we give to travellers? - Interim recommendations January 2020, from the Latin-American society for Travel Medicine (SLAMVI). Travel Med 
Infect Dis [Internet]. 2020 [acesso em 2020 Ago 13];33:101567. Disponível em: https://linkinghub.elsevier. com/retrieve/pii/S147789392030017X

5. Ministério da Saúde (BR), Secretaria de Gestão do Trabalho e da Educação na Saúde, Departamento de Gestão da Educação na Saúde Brasil. Residência Multiprofissional em Saúde: experiências, avanços e desafios. Brasília: Ministério da Saúde; 2006.

6. Tavares LRC, Costa JLR, Oishi J, Driusso P. Inserção da fisioterapia na atenção primária à saúde: análise do cadastro nacional de estabelecimentos de saúde em 2010. Fisioter Pesqui [Internet]. 2018 [acesso em 2020 Ago 13];25(1):9-19. Disponível em: http://www.scielo.br/scielo.php?script=sci_arttext\&pid=S180929502018000100009\&lng=pt\&tlng=pt

7. Ministério da Saúde (Brasília). Protocolo de manejo clínico da Covid-19 na Atenção Primária à saúde: versão 9 [Internet]. 9th ed. 2020 [acesso em 2020 Ago 13]. Disponível em: https://portaldeboaspraticas.iff.fiocruz.br/ biblioteca/protocolo-de-manejo-clinico-do-coronavirus-covid-19-na-atencao-primaria-a-saude/

8. Vitória AM, Campos GWS. Só com APS forte o sistema pode ser capaz de achatar a curva de crescimento da pandemia e garantir suficiência de leitos UTI. Frente Estamira CAPS [Internet]. 2020 [acesso em 2020 Ago 13];25(1). Disponível em: https://frenteestamira.org/2020/04/02/so-com-aps-forte-o-sistema-pode-ser-capazde-achatar-a-curva-de-crescimento-da-pandemia-e-garantir-suficiencia-de-leitos-uti/

9. Ministério da Saúde (BR). Política Nacional de Promoção da Saúde [Internet]. 3rd ed. 2010 [acesso em 2020 Ago 13]. Disponível em: https://bvsms.saude.gov.br/bvs/publicacoes/politica_nacional_promocao_saude_3ed. pdf

10. Oliveira WK. Plano de Contingência Nacional para Infecção Humana pelo novo Coronavírus COVID-19 [Internet]. Brasília: Ministério da Saúde; 2020 [acesso em 2020 Ago 13]. Disponível em: www.saude.gov.br/ bvs\%0Ahttps://portalarquivos2.saude.gov.br/images/pdf/2020/fevereiro/13/plano-contingencia-coronavirusCOVID19.pdf

11. Ferreira SC. Sobre a eficiência de barreiras sanitárias restritivas para conter o avanço da COVID-19: uma modelagem matemática simples [Internet]. São Paulo: UPS; 2020 [acesso em 2020 Ago 13]. doi: 0000-0001$7159-2769$

12. Governo do Estado de Minas Gerais. Orientações sobre medidas de restrição de locomoção e Barreiras Sanitárias locais no Estado [Internet]. 2020 [acesso em 2020 Ago 13]. Disponível em: https://www.cosemsmg. org.br/site/Arquivos/PDF/notacoesbarreira.pdf

13. Governo do Estado de Mato Grosso do Sul. Manual de orientações: manejo clínico dos pacientes suspeitos e confirmados de Covid-19 [Internet]. 2nd ed. 2020 [acesso em 2020 Ago 13]. Disponível em: https://www. coronavirus.ms.gov.br/wp-content/uploads/2020/05/Manejo-Clínico-de-Pacientes-suspeitos-e-confirmadosde-Covid-19-1.pdf

14. Governo de Santa Catarina. Orientações para prevenção de contágio por Coronavírus (Covid-19) em alojamento para hospedagem temporária de trabalhadores sob responsabilidade do empregador [Internet]. 2017 [acesso em 2020 Ago 13]. Disponível em: https://www.saude.sc.gov.br/coronavirus/arquivos/ntc-0102020.PDF

15. Jackson JM Filho, Assunção AÁ, Algranti E, Garcia EG, Saito CA, Maeno M. A saúde do trabalhador e o enfrentamento da COVID-19. Rev Bras Saúde Ocup [Internet]. 2020 [acesso em 2020 Ago 13];45. Disponível em: http://www.scielo.br/scielo.php?script=sci_arttext\&pid=S0303-76572020000100100\&tlng=pt

16. Lourenço BS, Peres MAA, Porto IS, Oliveira RMP, Dutra VFD. Atividade física como uma estratégia terapêutica em saúde mental: revisão integrativa com implicação para o cuidado de enfermagem. Esc Anna Nery [Internet]. 2017 [acesso em 2020 Ago 13];21(3):1-8. Disponível em: http://www.scielo.br/scielo. php?script=sci_arttext\&pid=\$1414-81452017000300801\&lng=en\&tlng=en

17. Santos NC, Santos LS, Camelier FWR, Maciel RRBT, Portella DDA. Tecnologias aplicadas à promoção da saúde do trabalhador: uma revisão sistemática. Rev Bras Med do Trab [Internet]. 2017 [acesso em 2020 Ago 13 n];15(1):113-22. Disponível em: http://www.rbmt.org.br/details/219/pt-BR/tecnologias-aplicadas-apromocao-da-saude-do-trabalhador--uma-revisao-sistematica 
18. Machado AGM, Wanderley LCS. Educação em Saúde [Internet]. 2009 [acesso em 2020 Ago 13]. Disponível em: https://www.unasus.unifesp.br/biblioteca_virtual/esf/2/unidades_conteudos/unidade09/unidade09.pdf

19. Associação Brasileira de Empresas de Limpeza Pública e Resíduos Especiais. Recomendações para a Gestão de Resíduos Sólidos Durante a Pandemia de Coronavírus (Covid-19) [Internet]. 2020 [acesso em 2020 Ago 13]. Disponível em: https://abrelpe.org.br/abrelpe-no-combate-a-covid-19/

20. Takayanagui AMM, Santos CV, Souza RMGL. Gerenciamento dos resíduos gerados nos cuidados com a Covid-19 nos domicílios [Internet]. São Paulo: ABES-SP; 2020 [acesso em 2020 Ago 13]. Disponível em: https://www.sigam.ambiente.sp.gov.br/sigam3/repositorio/506/documentos/gerencimento_residuos_covid19. pdf

21. Melo MCH, Cruz GDC. Roda de conversa: uma proposta metodológica para a construção de um espaço de diálogo no Ensino Médio. Imagens Educ [Internet]. 2014 [acesso em 2020 Ago 13];4(2):31. Disponível em: http://periodicos.uem.br/ojs/index.php//magensEduc/article/view/22222

22. Inojosa R. Sinergia em políticas e serviços públicos: desenvolvimento social com intersetorialidade. Cadernos Fundap. 2011;22:102-10.

23. Ministério da Saúde (BR), Secretaria de Vigilância em Saúde. Política Nacional de Promoção da Saúde. Brasília: Ministério da Saúde; 2006.

24. Ministério da Saúde (BR). Nota técnica: atenção a pessoas com doenças crônicas na APS diante a situação de Pandemia de Covid-19 (Coronavírus). Brasília: Ministério da Saúde; 2020.

25. Sales RDC. O papel do fisioterapeuta residente multiprofissional em saúde da família: um relato de experiência. Rev APS [Internet]. 2017 [acesso em 2020 Ago 13];19(3):500-4. Disponível em: https://aps.ufjf. emnuvens.com.br/aps/article/view/2301/1028

26. Barbosa VBA, Ferreira MLSM, Barbosa PMK. Educação permanente em saúde: uma estratégia para a formação dos agentes comunitários de saúde. Rev Gaúcha Enferm [Internet]. 2012 [acesso em 2020 Ago 13];33(1):56-63. Disponível em: http://www.scielo.br/scielo.php?script=sci_arttext\&pid=S198314472012000100008\&lng=pt\&tlng=pt

27. Queiroz DM, Silva MRF, Oliveira LC. Educação permanente com agentes comunitários de saúde: potencialidades de uma formação norteada pelo referencial da Educação Popular e Saúde. Interface Comun Saúde Educ [Internet]. 2014 [acesso em 2020 Ago 13];18(suppl 2):1199-210. Disponível em: http://www. scielo.br/scielo.php?script=sci_arttext\&pid=S1414-32832014000601199\&lng=pt\&tIng=pt

28. Ministério da Saúde (BR). Recomendações para adequação das ações dos agentes comunitários de saúde frente à atual situação epidemiológica referente ao covid-19 Brasília/DF [Internet]. 2020 [acesso em 2020 Ago 13]. Disponível em: http://189.28.128.100/dab/docs/portaldab/documentos/20200324_recomendacoes_ACS_ COVID19_ver001_final.pdf

29. Catavi H Sobrinho, Silva LMF, Porto BS. Comunicação dialógica e ciência da informação: modelo para a organização e representação do conhecimento. ISKO Bras [Internet]. 2019 [acesso em 2020 Ago 13];6(120810-20191003112057):19-27. Disponível em: https://brapci.inf.br/index.php/res/v/123168

30. Secretaria de Estado de Saúde de Minas Gerais. Informe epidemiológico coronavírus [Internet]. 2020 [acesso em 2020 Ago 13]. Disponível em: https://www.saude.mg.gov.br/component/gmg/story/12686-informeepidemiologico-coronavirus-13-05-2020

31. Ministério da Saúde (BR), Secretaria de Atenção Primária à Saúde, Departamento de Saúde da Família Brasil. Núcleo Ampliado de Saúde da Família e Atenção Básica (NASF-AB) e Programa Previne Brasil [Internet]. 2020 [acesso em 2020 Ago 13]. Disponível em: http://189.28.128.100/dab/docs/portaldab/ documentos/NT_NASF-AB_Previne_Brasil.pdf 


\section{Endereço para correspondência:}

Eduardo Augusto Barbosa Figueiredo

Universidade Federal dos Vales do Jequitinhonha e Mucuri

Rodovia MGT 367, Km 583, 5000

Bairro: Alto da Jacuba

CEP: 39100-000 - Diamantina - MG - Brasil

E-mail: eduuabf@gmail.com

Como citar: Figueiredo EAB, Silva KLS, Luz HDH, Martins FLM, Marçal MA, Vitorino DFM, et al. Atuação do Fisioterapeuta na saúde primária no enfrentamento da COVID 19: relato de experiência. Rev Bras Promoç Saúde. 2021;34:11164. 